\title{
Constraints on neutrino masses from leptogenesis models
}

\author{
Thomas Hambye ${ }^{a}$, Yin $\operatorname{Lin}^{b}$, Alessio Notari ${ }^{b}$, \\ Michele Papucci ${ }^{b}$ and Alessandro Strumia ${ }^{c}$ \\ ${ }^{a}$ Department of Physics, Theoretical Physics, University of Oxford, Oxford OX1 3NP, UK \\ ${ }^{b}$ Scuola Normale Superiore, Piazza dei Cavalieri 7, I-56126 Pisa and INFN, Italia \\ ${ }^{c}$ Dipartimento di Fisica dell'Università di Pisa and INFN, Italia
}

\begin{abstract}
Upper bounds on the CP asymmetry relevant for leptogenesis are reexamined and found weaker than in previous literature, both for hierarchical and for quasi-degenerate righthanded neutrinos. Successful leptogenesis implies the usual lower bound on right-handed neutrino masses, and an upper bound on left-handed neutrino masses (which we obtain to be $0.15 \mathrm{eV}$ at $3 \sigma$ ) only if right-handed neutrinos are assumed to be much more hierarchical than left-handed neutrinos. Otherwise both bounds can be considerably relaxed. The constraint on light neutrino masses varies assuming different interpretations of why neutrinos should be quasi-degenerate. With conservative assumptions, we find that a mild quasi-degeneracy allows neutrinos heavier than an eV compatibly with leptogenesis.

We also extend computations of thermal leptogenesis to an alternative model of neutrino mass mediated by fermion triplets which was never considered so far for leptogenesis. Leptogenesis can be successful despite the effect of gauge interactions, resulting in only slightly stronger constraints on neutrino masses.
\end{abstract}

Assuming that neutrino masses are generated by tree level exchange of right-handed neutrinos, that the observed baryon asymmetry is produced via thermal leptogenesis 1 and that right-handed neutrinos are hierarchical, one can derive interesting constraints [2, 3, 4]: right-handed neutrinos must be heavier than about $10^{8} \mathrm{GeV}$ and left-handed neutrinos must be lighter than about $0.1 \mathrm{eV}$ 3. 4. Since the former constraint leads to a possible conflict between leptogenesis and gravitino overproduction, and since the later one is stronger than present experimental bounds and is close to the mass scale suggested by atmospheric oscillations (i.e. $m_{3} \gtrsim 0.05 \mathrm{eV}$ ), in this article we reconsider these constraints in details. To this end we adopt the results of 4 for a precise computation of the dynamics of thermal leptogenesis, and we reexamine the upper bound on $\mathrm{CP}$ violation in right-handed neutrino decays.

In section 1 we consider a hierarchical spectrum of right-handed neutrinos. We show that the lower bound above on their masses can be significantly evaded dropping the assumption (made in [2]) that the hierarchy among right-handed neutrinos is much larger than the observed one among lefthanded neutrinos. Moreover, even under this assumption, we derive a precise upper bound on the CP-asymmetry and find it weaker than in previous literature [3], leading to a slightly higher neutrino mass bound.

Since this constraint is based on the doubtful assumption that quasi-degenerate neutrinos be produced by hierarchical right-handed neutrinos, in section 2 we study what happens allowing quasidegenerate right-handed neutrinos. As well known, the asymmetry can be resonantly enhanced [5], 6]; with respect to the analysis of [3] we find other effects that relax the upper bound on the CP-asymmetry so that our constraint on neutrino masses is much weaker. We discuss how the result depends on possible reasons that naturally give rise to quasi-degenerate left-handed neutrinos. 
In section 3 we study alternative mechanisms of leptogenesis and discuss the corresponding constraints on neutrino masses. Neutrino masses can be produced by tree-level exchange of three different kinds of particles: a) right-handed neutrinos [7; b) fermion $\mathrm{SU}(2)_{L}$ triplets [8, 9]; c) one or more scalar triplets [10]. Special emphasis is put on the case b) which has never been considered for leptogenesis. We show that it is efficient, even if in this case the gauge interactions can keep triplets close to thermal equilibrium.

Results are summarized in section 4.

\section{Hierarchical right-handed neutrinos}

If neutrino masses are produced by the see-saw model described by the following Lagrangian

$$
\mathscr{L}=\mathscr{L}_{\mathrm{SM}}+\bar{N}_{i} i \not \partial N_{i}+\left(\lambda^{i j} N^{i} L^{j} H+\frac{M_{N}^{i j}}{2} N_{i} N_{j}+\text { h.c. }\right)
$$

the most generic high energy parameters that give rise to the desired neutrino masses $m_{3}>m_{2}>m_{1} \geq$ 0 and mixings $V$ can be written as [1]

$$
M_{N}=\operatorname{diag}\left(M_{1}, M_{2}, M_{3}\right), \quad \lambda=\frac{1}{v} M_{N}^{1 / 2} \cdot R \cdot \operatorname{diag}\left(m_{1}, m_{2}, m_{3}\right)^{1 / 2} \cdot V^{\dagger} .
$$

One can always work in the mass eigenstate basis of right-handed neutrinos, and choose $M_{3}>M_{2}>$ $M_{1} \geq 0 . R$ is an arbitrary complex orthogonal matrix (i.e. $R^{T} \cdot R=1$ ), that can be written in terms of 3 complex mixing angles as

$$
R=\operatorname{diag}( \pm 1, \pm 1, \pm 1) R^{(23)}\left(z_{23}\right) R^{(13)}\left(z_{13}\right) R^{(12)}\left(z_{12}\right)
$$

where $R^{(i j)}$ is a rotation in the $(i j)$ plane with complex angles $z_{i j}$. This parameterization explicitly shows that the see-saw model has 12 real and 6 complex parameters beyond ones already present in the SM: $6+3$ can be measured by low energy experiments $\left(m_{1}, m_{2}, m_{3}\right.$ and the three complex mixing angles in $V$ ) while the remaining $6+3\left(M_{1}, M_{2}, M_{3}\right.$ and the three complex mixing angles in $\left.R: z_{12}, z_{23}, z_{13}\right)$ cannot.

\section{The CP asymmetry}

One important ingredient that determines the baryon asymmetry produced by thermal leptogenesis is the CP asymmetry $\varepsilon_{i}$ in decays of right-handed neutrinos $N_{i}$. Since we will later study the generic case where right-handed neutrinos can be quasi-degenerate, it is useful to decompose the CP-asymmetry in $N_{1}$ decays (and similarly for $N_{2,3}$ decays) as the sum of a $V$ ertex contribution and of a Self-energy contribution (with the self energy contribution as given in [6])

$$
\varepsilon_{1}=-\sum_{j=2,3} \frac{3}{2} \frac{M_{1}}{M_{j}} \frac{\Gamma_{j}}{M_{j}} I_{j} \frac{2 S_{j}+V_{j}}{3}
$$

where

$$
I_{j}=\frac{\operatorname{Im}\left[\left(\lambda \lambda^{\dagger}\right)_{1 j}^{2}\right]}{\left|\lambda \lambda^{\dagger}\right|_{11}\left|\lambda \lambda^{\dagger}\right|_{j j}}, \quad \frac{\Gamma_{j}}{M_{j}}=\frac{\left|\lambda \lambda^{\dagger}\right|_{j j}}{8 \pi} \equiv \frac{\tilde{m}_{j} M_{j}}{8 \pi v^{2}}
$$

and where

$$
S_{j}=\frac{M_{j}^{2} \Delta M_{1 j}^{2}}{\left(\Delta M_{1 j}^{2}\right)^{2}+M_{1}^{2} \Gamma_{j}^{2}}, \quad V_{j}=2 \frac{M_{j}^{2}}{M_{1}^{2}}\left[\left(1+\frac{M_{j}^{2}}{M_{1}^{2}}\right) \log \left(1+\frac{M_{1}^{2}}{M_{j}^{2}}\right)-1\right],
$$

are loop factors, with $\Delta M_{i j}^{2}=M_{j}^{2}-M_{i}^{2}$. In the parameterization of eq. (2), the light neutrino mixing matrix $V$ does not affect $\varepsilon_{1}$ and CP-violation in leptogenesis arises from $R$. While $V_{j} \leq 1$, the factor $S_{j}$ is resonantly enhanced, up to $S_{j} \sim M_{j} / \Gamma_{j}$, when $M_{j}-M_{1} \sim \Gamma_{j}$. We normalized the resonance factors 

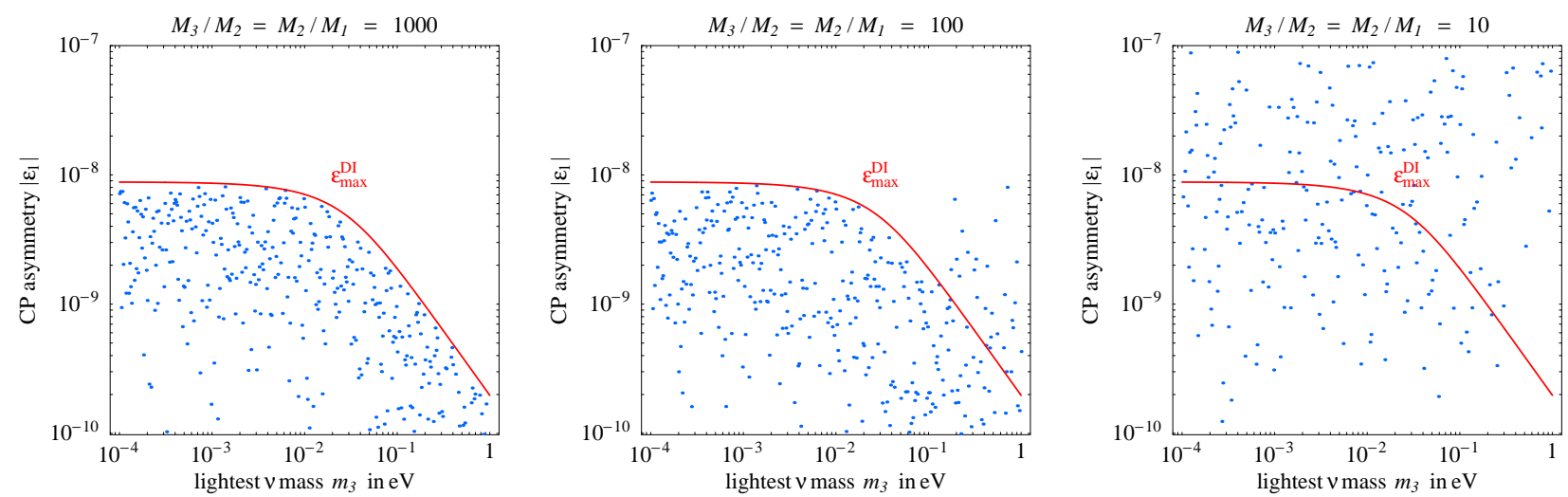

Figure 1: The Davidson-Ibarra bound on the CP asymmetry $\varepsilon_{1}$ was derived and holds for $M_{2,3} / M_{1}=\infty$. The random samplings of the parameter space (performed assuming different finite hierarchies among right-handed neutrinos) confirms that $\left|\varepsilon_{1}\right|$ can be above the DI bound. For all points $\left|\lambda_{i j}\right|<\sqrt{4 \pi}$, $\Delta m_{\mathrm{atm}}^{2}=210^{-3} \mathrm{eV}^{2}, M_{1}=10^{8} \mathrm{GeV}$. As usual, the density of points depends on arbitrary details of the sampling procedure and carries no information about the likelihood of different regions.

$S_{j}$ and the vertex factors $V_{j}$ in such a way that $S_{2,3}=1$ and $V_{2,3}=1$ (so that $\left(2 S_{j}+V_{j}\right) / 3=1$ ) in the hierarchical limit $M_{2,3} / M_{1} \rightarrow \infty$. In this limit, inserting the parameterization of eq. (2) into eq. (5) gives

$$
\varepsilon_{1}=-\sum_{j=2,3} \frac{3}{16 \pi} \frac{M_{1}}{M_{j}} \frac{\operatorname{Im}\left[\left(\lambda \lambda^{\dagger}\right)_{1 j}^{2}\right]}{\left|\lambda \lambda^{\dagger}\right|_{11}}=-\frac{3}{16 \pi} \frac{M_{1}}{v^{2}} \frac{\sum_{i} m_{i}^{2} \operatorname{Im} R_{1 i}^{2}}{\sum_{i} m_{i}\left|R_{1 i}\right|^{2}},
$$

which leads to the Davidson-Ibarra (DI) bound [2]:

$$
\left|\varepsilon_{1}\right| \leq \varepsilon_{\max }^{\mathrm{DI}}=\frac{3}{16 \pi} \frac{M_{1}}{v^{2}}\left(m_{3}-m_{1}\right),
$$

where $m_{3}\left(m_{1}\right)$ is the mass of the heaviest (lightest) neutrino. The DI upper bound is plotted in fig. 11. together with a random sampling that turns out to find points above it. In fact, the DI bound is derived and holds for $M_{2,3} / M_{1}=\infty$ while in fig. 1 we assumed a finite hierarchy. One expects that for $M_{2,3} \gg M_{1}$ the DI bound remains approximatively valid, up to small corrections of relative order $\left(M_{1} / M_{2,3}\right)^{2}$. We now explain why this is not the case.

In the infinitely hierarchical limit $\varepsilon_{1}$ is given by a sum over right-handed neutrinos weighted by $1 / M_{j}$ exactly like neutrino masses: the resulting simple expression has special properties, e.g. in this limit $\mathrm{CP}$ violation in leptogenesis disappears when light neutrinos are degenerate. The extra terms suppressed by $\left(M_{1} / M_{2,3}\right)^{2}$ do not share this property: e.g. they do not vanish when neutrinos are degenerate. Moreover, even with hierarchical neutrinos, these extra terms can be enhanced by $\tilde{m}_{2,3} / m_{2,3}$. One gets points above the DI bound when the enhancements overcompensate the $\left(M_{1} / M_{2,3}\right)^{2}$ suppression.

This observation can be relevant for leptogenesis, since this enhancement of $\varepsilon_{1}$ can be achieved without introducing significant wash-out factors in the dynamics of thermal leptogenesis. In fact, $\Delta L=2$ washout scatterings mediated by off-shell $N_{1,2,3}$ exchange are controlled by neutrino masses and do not depend on $\tilde{m}_{2,3}$ (and scatterings mediated by on-shell $N_{2,3}$ are Boltzmann suppressed). On the contrary $\Delta L=2$ scatterings mediated by on-shell $N_{1}$ exchange are controlled by $\tilde{m}_{1}$, and the efficiency of leptogenesis is maximal for a relatively small value of $\tilde{m}_{1} \sim 10^{-3} \mathrm{eV}[3$, 4]. Therefore we must show that the enhancement under discussion is possible for small $\tilde{m}_{1}$. We show this analytically and obtain simple estimates by focussing on the simple limit $m_{1}=m_{2}=0$. (Our final result remains valid in the more complicated realistic case with small non-zero $m_{1}$ and $m_{2}$ ). Inserting in the parameterization (2) $z_{23}=0+i y_{23}$ and $z_{13}=x_{13}+i y_{13}$ with $\cos 2 x_{13}=1 / \cosh 2 y_{13}$ we get

$$
\varepsilon_{1}=-\frac{3}{16 \pi} \frac{M_{1} m_{3}}{v^{2}}\left(F_{3} \cosh ^{2} y_{23}-F_{2} \sinh ^{2} y_{23}\right), \quad \tilde{m}_{1}=m_{3}\left|\sin z_{13}\right|^{2}=m_{3} \frac{\cosh 2 y_{13}-\cos 2 x_{13}}{2} .
$$


Choosing a small $x_{13}$ allows to keep $\tilde{m}_{1}$ arbitrarily small without affecting $\varepsilon_{1}$. In the fully hierarchical limit the loop functions $F_{i}=\left(2 S_{i}+V_{i}\right) / 3$ satisfy $F_{2}=F_{3}=1$ and the last term in $\varepsilon_{1}$ simplifies to 1 , giving $\left|\varepsilon_{1}\right|=\varepsilon_{\mathrm{DI}}^{\max }$. For finite $M_{1} / M_{2,3}$ one instead has $F_{2} \neq F_{3}$ and $\varepsilon_{1}$ can be enhanced by choosing a large $y_{23}$. The maximal value is limited only by perturbativity of the largest neutrino Yukawa coupling. This violation of the DI bound does not correspond to a local maximum of $\varepsilon_{1}$ and therefore was missed in analyses that tried to maximize $\varepsilon_{1}$ by imposing $d \varepsilon_{1} / d z_{i j}=0$. Imposing $\lambda_{33}=\cosh y_{23} \sqrt{M_{3} m_{3}} / v \lesssim \sqrt{4 \pi}$ gives

$$
\left|\varepsilon_{1}\right| \lesssim \max \left(\frac{M_{1}^{3}}{M_{3} M_{2}^{2}}, \varepsilon_{\max }^{\mathrm{DI}}\right)
$$

This estimate is confirmed by the random sampling in figure 1 performed for $M_{1}=10^{8} \mathrm{GeV}$ which is probably the most interesting choice, as the DI bound implies $M_{1} \gtrsim 10^{8} \mathrm{GeV}$ (see e.g. 4]). Allowing only neutrino Yukawa couplings smaller than ${ }^{1} \sqrt{4 \pi}$ and assuming $M_{3} / M_{2}=M_{2} / M_{1}=10^{n}$ the new configuration under discussion allows to reach $\left|\varepsilon_{1}\right| \lesssim 10^{-4 n}$. The DI bound was derived and holds in the limit $n \rightarrow \infty$. For $n=3$ the DI bound is still an excellent approximation (fig. 1 $\mathrm{a}$ ). For $n=2$ the DI bound starts failing only when neutrinos are quasi-degenerate (fig. 10). For $n=1$ the DI bound can be significantly evaded (fig. 1:). This is possibly the most realistic case, as solar and atmospheric oscillations indicate that there is at most a mild hierarchy between left-handed neutrinos: $m_{3} / m_{2} \lesssim 6$. Allowing right-handed neutrinos to have a similarly mild hierarchy, $M_{2} / M_{1} \sim 10$, eq. (10) shows that successful leptogenesis with hierarchical $N_{i}$ is possible even for $N_{1}$ much lighter than $10^{8} \mathrm{GeV}$, as possibly needed in supersymmetric models in order to avoid overproduction of gravitinos [12]. Thermal leptogenesis can be successful even if right-handed neutrinos are light and hierarchical. We can make a more quantitative statement by assuming a $10^{-3}$ efficiency (this is a reasonably conservative value, see e.g. (4): eq. (10) then requires $M_{3} M_{2}^{2} / M_{1}^{3} \lesssim 10^{4}$ and consequently allows some hierarchy among $M_{1,2,3}$.

However, a CP-asymmetry above the DI bound is realized for $\tilde{m}_{2,3} \gg m_{2,3}$ and therefore involves apparently unlikely cancellations, as it needs that $N_{2}$ and $N_{3}$ each gives a large contribution to neutrino masses, but they cancel among each others. This configuration seems justifiable in a natural way, by e.g. building models where $N_{2}$ and $N_{3}$ form a quasi-Dirac couple with quasi-chiral Yukawa couplings. See also the appendix of [13].

In the rest of this section we assume that $M_{3} M_{2}^{2} / M_{1}^{3}$ is large enough for the DI upper bound to hold and precisely compute, under all stated assumptions, the maximal value of neutrino masses compatible with thermal leptogenesis. We find that previous bounds must be weakened for a different reason, which has no relation with the above discussion.

\section{Bound on neutrino masses}

The DI bound becomes more stringent if neutrinos are quasi-degenerate since in this case $m_{3}-m_{1} \simeq$ $\Delta m_{\mathrm{atm}}^{2} / 2 m_{3}$ in eq. (8) decreases. Moreover in this case the efficiency factor $\eta$ is smaller because larger neutrino masses need larger neutrino Yukawa couplings and therefore implies larger wash-out scattering rates. In fact the out-of equilibrium condition $\Gamma \lesssim H\left(M_{1}\right)$ means $\tilde{m}_{1} \lesssim 10^{3} v^{2} / M_{\mathrm{Pl}} \sim 10^{-3} \mathrm{eV}$ where

$$
\left.\tilde{m}_{1} \equiv\left|\lambda \lambda^{\dagger}{ }_{11} \frac{v^{2}}{M_{1}}=8 \pi \Gamma_{1} \frac{v^{2}}{M_{1}^{2}}=\sum_{i} m_{i}\right| R_{1 i}\right|^{2}
$$

is always larger than $m_{1}$. The minimum value $\tilde{m}_{1}=m_{1}$ implies $R_{12}=R_{13}=0$ and therefore a vanishing CP-asymmetry (in the $M_{2,3} / M_{1}=\infty$ limit). Conversely, the DI bound is saturated for large values of $\tilde{m}_{1}$, when $\eta$ is strongly suppressed. As a result the maximal baryon asymmetry is reached for $\tilde{m}_{1}$ larger than $m_{1} \approx m_{3}$ but rather close to it $\underline{3}$.

In order to compute the leptogenesis constraint on neutrino masses, one must compute the maximal value of $\varepsilon_{1}$ at given $\tilde{m}_{1}$, and next maximize the baryon asymmetry $n_{B} / n_{\gamma} \approx 0.01 \varepsilon_{1} \eta$ with respect to

\footnotetext{
${ }^{1}$ In supersymmetric models where sleptons acquire SUSY-breaking mass terms at high scale the non-observation of $\mu \rightarrow e \gamma$ implies a somewhat stronger bound, $\left|\lambda^{\dagger} \lambda\right|_{e \mu} \lesssim 10^{-1 \pm 1}$.
} 


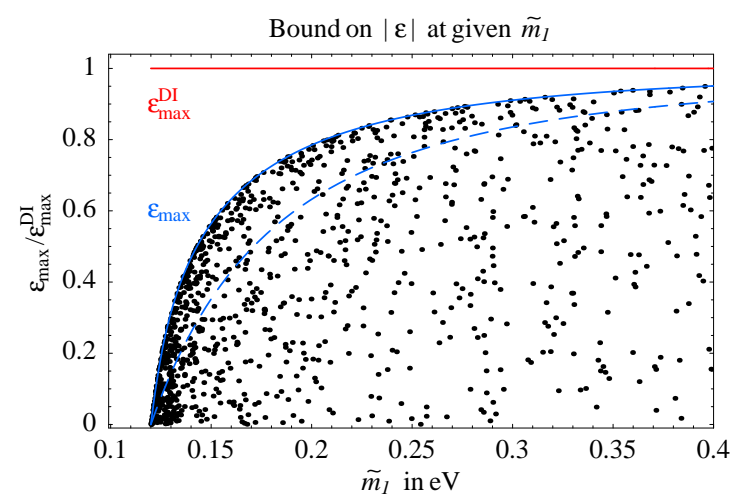

Figure 2: Maximal value of $\varepsilon_{1}$ assuming a large hierarchy at fixed $\tilde{m}_{1}$. The dashed line shows the attempt in [3]. The random sampling was performed as an additional check that the analytical bound of eq. (11) is correct.

remaining free parameters, essentially $\tilde{m}_{1}$ and $M_{1}$. To determine the bound on $\varepsilon_{1}$ at fixed $\tilde{m}_{1}$ we can neglect $\Delta m_{\text {sun }}^{2} \ll \Delta m_{\text {atm }}^{2}$, so that $m_{1}=m_{2}$ and we end up with a 2 neutrino case: rotations in the (12) plane do not have physical effects. ${ }^{2}$ We can write $R$ as

$$
R=R^{(13)}\left(z_{13}\right)=\left(\begin{array}{ccc}
\cos z_{13} & 0 & \sin z_{13} \\
0 & 1 & 0 \\
-\sin z_{13} & 0 & \cos z_{13}
\end{array}\right)
$$

because $\varepsilon_{1}$ does not depend on $z_{23}$. Here $z_{13}=x+i y$ is a complex mixing angle. The condition $\tilde{m}_{1}=m_{1}\left|R_{11}\right|^{2}+m_{3}\left|R_{13}\right|^{2}$ fixes $x$ as function of $y$ :

$$
\cos 2 x=\frac{2 \tilde{m}_{1}-\left(m_{1}+m_{3}\right) \cosh 2 y}{m_{3}-m_{1}},
$$

allowing to write the CP asymmetry as

$$
\left|\varepsilon_{1}\right|=\frac{3}{16 \pi} \frac{M_{1}}{v^{2}} \frac{m_{3}^{2}-m_{1}^{2}}{\tilde{m}_{1}}\left|\operatorname{Im} R_{11}^{2}\right|=\frac{3}{16 \pi} \frac{M_{1}}{v^{2}} \frac{m_{3}^{2}-m_{1}^{2}}{2 \tilde{m}_{1}} \sinh 2 y \sqrt{1-\left(\frac{2 \tilde{m}_{1}-\left(m_{1}+m_{3}\right) \cosh 2 y}{m_{3}-m_{1}}\right)^{2}} .
$$

Maximizing with respect to $y$ gives our bound:

$$
\left|\varepsilon_{1}\right| \leq \varepsilon_{\max }=\frac{\varepsilon_{\mathrm{DI}}^{\max }}{2} \sqrt{1-\left[(1-a) \tilde{m}_{1} /\left(m_{3}-m_{1}\right)\right]^{2}} \sqrt{(1+a)^{2}-\left[\left(m_{3}+m_{1}\right) / \tilde{m}_{1}\right]^{2}},
$$

where

$$
a=2 \operatorname{Re}\left[\frac{m_{1} m_{3}}{\tilde{m}_{1}^{2}}\right]^{1 / 3}\left[-1-i \sqrt{\frac{\left(m_{1}^{2}+m_{3}^{2}+\tilde{m}_{1}^{2}\right)^{3}}{27 m_{1}^{2} m_{3}^{2} \tilde{m}_{1}^{2}}-1}\right]^{1 / 3}>0 .
$$

In the hierarchical and quasi-degenerate light neutrino limits it simplifies to

$$
\left|\varepsilon_{1}\right| \leq \varepsilon_{\max } \simeq \varepsilon_{\mathrm{DI}}^{\max } \times \begin{cases}1-m_{1} / \tilde{m}_{1} & \text { if } m_{1} \ll m_{3} \\ \sqrt{1-m_{1}^{2} / \tilde{m}_{1}^{2}} & \text { if } m_{1} \simeq m_{3}\end{cases}
$$

The continuous line in fig. 2 (plotted assuming $m_{1}=0.12 \mathrm{eV}$ ) is our upper bound. The dashed line shows the result of 3 , that first attempted to compute $\varepsilon_{\max }$. Their bound in the quasi-degenerate limit

\footnotetext{
${ }^{2}$ One can explicitly verify that they do not affect $\varepsilon_{1}$. From its explicit expression in eq. (7) the matrix $R_{12}$ in $\lambda$ commutes with diag $\left(m_{1}, m_{2}, m_{3}\right)$ and cancels out with $R_{12}^{T}$ coming from $\lambda^{T}$. This holds for the numerator of eq. (7) because we assumed $M_{2,3} / M_{1}=\infty$ and will be no longer true when we will relax this assumption. The denominator has a dependence on $\operatorname{Im}\left(z_{12}\right)$, but only as $c+c^{\prime} \cosh \left[\operatorname{Im}\left(z_{12}\right)\right]$ with $c, c^{\prime}$ positive. The maximization of $\varepsilon_{1}$ naturally leads to $\operatorname{Im}\left(z_{12}\right)=0$, hence the validity of what stated.
} 

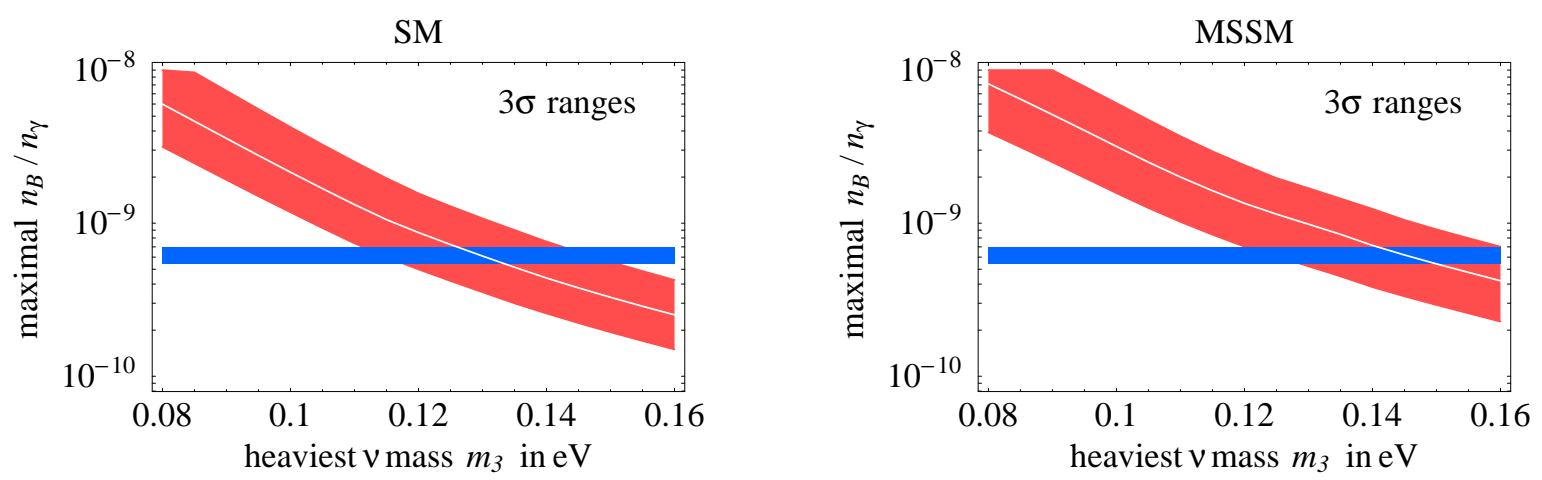

Figure 3: Leptogenesis constraint on neutrino masses assuming $M_{2,3} \gg M_{1}$. The plot shows the measured baryon asymmetry (horizontal line) compared with the maximal leptogenesis value as function of the heaviest neutrino mass $m_{3}$, renormalized at low energy. Error bars are at $3 \sigma$.

reduces to $\varepsilon_{\mathrm{DI}}^{\max }\left(1-m_{1}^{2} / \tilde{m}_{1}^{2}\right)$ and would be stronger than our bound, but does not hold as confirmed by the numerical scanning. ${ }^{3}$ In the hierarchical limit $m_{1} \ll m_{3}$ the difference becomes less relevant. In the MSSM the CP-asymmetry, and consequently its upper limit, is 2 times larger than in the SM. The MSSM result in fig. 3 is is obtained using the Boltzmann equations of [4.

Combining our revised bound on the CP-asymmetry with the revised computation of the efficiency of leptogenesis of $[4]^{4}$ we get

$$
m_{3}<0.15 \mathrm{eV} \quad(\text { at } 3 \sigma)
$$

as illustrated in fig. 3. This constraint holds under the doubtful assumption that hierarchical righthanded neutrinos give quasi-degenerate left-handed neutrinos.

\section{Quasi-degenerate right-handed neutrinos}

Successful thermal leptogenesis implies interesting restrictions on the masses of quasi-degenerate neutrinos under the hypothesis of hierarchical right-handed neutrinos. This is a crucial but doubtful assumption. In fact, one expects that quasi-degenerate neutrinos be more naturally produced by quasi-degenerate right-handed neutrinos (rather than by an interplay between heavier $N_{2,3}$ with bigger Yukawa couplings and lighter $N_{1}$ with smaller couplings). In absence of simple predictive models one might naïvely expect that left-handed and right-handed neutrinos show similar levels of degeneracy.

In this section we study how much the constraint on neutrino masses gets relaxed when we make this kind of 'reasonable' assumptions. We think this is an interesting but qualitative issue. Therefore (unlike in the hierarchical case) we do not attempt to derive a precise absolute constraint. Our results should however be a qualitatively correct approximation to it. A fully precise discussion is anyhow prevented by the fact that, in a generic see-saw model, quasi-degenerate neutrinos are not even stable under radiative corrections.

We start studying simple particular cases and later show that they catch the new relevant effects that we need to consider.

\footnotetext{
${ }^{3}$ We explain analytically the reason of the disagreement. Ref. [3] made unjustified assumptions on the elements of $R$. In their notation variables $x_{i}$ and $y_{i}$ were used (with $\left.i=1,2,3\right)$, together with the correspondence: $R_{1 i}^{2} m_{i} /\left(\lambda \lambda^{\dagger}\right)_{11} \equiv x_{i}+i y_{i}$. The maximal $\left|\varepsilon_{1}\right|$ is reached for maximal $y_{3}$. In order to find this maximum they assume $x_{2}=y_{2}=x_{3}=0$. The first two assumptions are correct in the limit $\Delta m_{\text {sun }}^{2} \ll \Delta m_{\text {atm }}^{2}$ and correspond to our $z_{12}=0$. But the extra assumption $x_{3}=0$ (which would corresponds to $\operatorname{Re} R_{13}^{2}=0$ ) is incorrect, and doing so one does not get the true maximum. In our numerical example with quasi-degenerate neutrinos the maximum is reached for $x_{3} \approx 0.4$.

${ }^{4}$ In appendix $\mathrm{A}$ we explain why, when computing such constraints, the full network of Boltzmann equations can be approximated with a single equation for the total $B-L$ asymmetry, as tacitly assumed in previous analyses.
} 


\section{The conservative case}

We first study what happens for $M_{3} \gg M_{2} \sim M_{1}$. Using eq. (4), in this limit $\varepsilon_{1}$ (and similarly $\varepsilon_{2}$ ) can be approximated as

$$
\left|\varepsilon_{1}\right|=\frac{M_{1}}{M_{2}} \frac{\Gamma_{2}}{M_{2}} S_{2}\left|I_{2}\right|<\frac{1}{2} \frac{M_{1}}{M_{2}}\left|I_{2}\right| \simeq \frac{1}{2}\left|I_{2}\right|
$$

where the inequality is obtained by taking the resonance condition $M_{2}-M_{1}=\Gamma_{2} / 2$ which maximizes the resonance factor $S_{2}$ and gives $S_{2}=M_{2} / 2 \Gamma_{2}$. It is useful to compare eq. (14) with eq. (71). The DI upper bound in eq. (8) can be rewritten schematically as the product of two suppression factors,

$$
\frac{3}{2} \frac{M_{1}}{M_{j}} \frac{\Gamma_{j}}{M_{j}} \quad \text { times } \quad \frac{m_{3}-m_{1}}{\tilde{m}_{j}}
$$

present for different physical reasons. The first factor is related to the fact that heavy particles with small couplings give small effects and it is just the result of a naïve estimation of $\varepsilon_{1}$ performed dropping the flavour indices:

$$
\varepsilon_{1} \sim \frac{3}{16 \pi} \frac{M_{1}}{M_{j}} \frac{\lambda_{j}^{2} \lambda_{1}^{2}}{\lambda_{1}^{2}} \sim \frac{3}{2} \frac{M_{1}}{M_{j}} \frac{\Gamma_{j}}{M_{j}} .
$$

The second factor comes from a flavour subtelty. In the hierarchical limit, due to the orthogonality of the $R$ matrix in eq. (2), the $\lambda_{j}^{2}$ in the numerator of the asymmetry is not proportional to $\Gamma_{j}$ (or to $\tilde{m}_{j}$ ) as in eq. (16) but to the $m_{3}-m_{1}$ mass difference. This results in the extra $\left(m_{3}-m_{1}\right) / \tilde{m}_{j}$ suppression of the asymmetry, which is significant when neutrinos are quasi-degenerate. For example for $m_{1} \sim 1 \mathrm{eV}$ this suppression is at least of order $\left[\Delta m_{\mathrm{atm}}^{2} /\left(m_{3}+m_{1}\right)\right] / \tilde{m}_{j} \sim 10^{-3}$.

Eq. (14) shows that, when also right-handed neutrinos are quasi-degenerate, none of these two suppressions are there. The first one can be completely compensated by the resonance factor $S_{2}$ which is 1 in the hierarchical case and $M_{2} / 2 \Gamma_{2}$ at the resonance. The second suppression disappears because $\varepsilon_{1}$ is no longer directly related to neutrino masses, so that it no longer vanishes when neutrinos are degenerate. More technically, $I_{2}$ (unlike eq. (7)) is not suppressed by an orthogonality relation coming from the $R$ matrix.

The net result is that the $\mathrm{CP}$ asymmetry can be of order unity independently on the magnitude of the neutrino masses. Whether it is of order unity is controlled by the size of $I_{2}$ in eq. (14). As shown in details in appendix $\mathbb{B} I_{2}$ can be easily of order unity except if $\tilde{m}_{i}$ are very close to their minimum values (where $\varepsilon_{1}$ vanishes).

A formula that correctly estimates the maximal CP asymmetry not only in the quasi-degenerate case we are considering but also in the hierarchical limit and in intermediate cases is obtained by multiplying our bound $\varepsilon_{\max }$ of eq. (11) valid in the hierarchical limit with an appropriate rescaling factor:

$$
\left|\varepsilon_{1}\right| \lesssim \varepsilon_{\max } \frac{S_{2} m_{3}-m_{1}}{m_{3}-m_{1}} .
$$

This bound reduces to eq. (11) in the hierarchical limit $m_{3} \gg m_{1}$. In the quasi-degenerate limit, $m_{3} \simeq m_{1}$, it reproduces eq. (14) up to an order-one factor. This is the maximal CP-asymmetry possible if a quasi-degeneracy in neutrino masses, $m_{1} \approx m_{2} \approx m_{3} \approx \tilde{m}_{1} \approx \tilde{m}_{2} \approx \tilde{m}_{3}$ arises accidentally, as can happen if no flavour symmetry acts on neutrinos. In such a case one expects that the three $\tilde{m}_{i}$ are equal only up to order-one factors. The resulting bound on $m_{\nu}$ is given by the solid line in fig. 4 4 . A numerical sampling performed including $\mathcal{O}(1)$ factors reveals that (17) can be reached, and in fact the most conservative bound would be even somewhat weaker. Already for a $\sim 10 \%$ degeneracy between $N_{2}$ and $N_{1}$ successful leptogenesis can occur for neutrinos heavier than $1 \mathrm{eV}$.

\section{A simple special limit}

Next we consider the case where the 3 right-handed neutrinos are quasi-degenerate. It is interesting to consider first the case where two neutrinos are exactly degenerate and quasi-degenerate to a third one, 

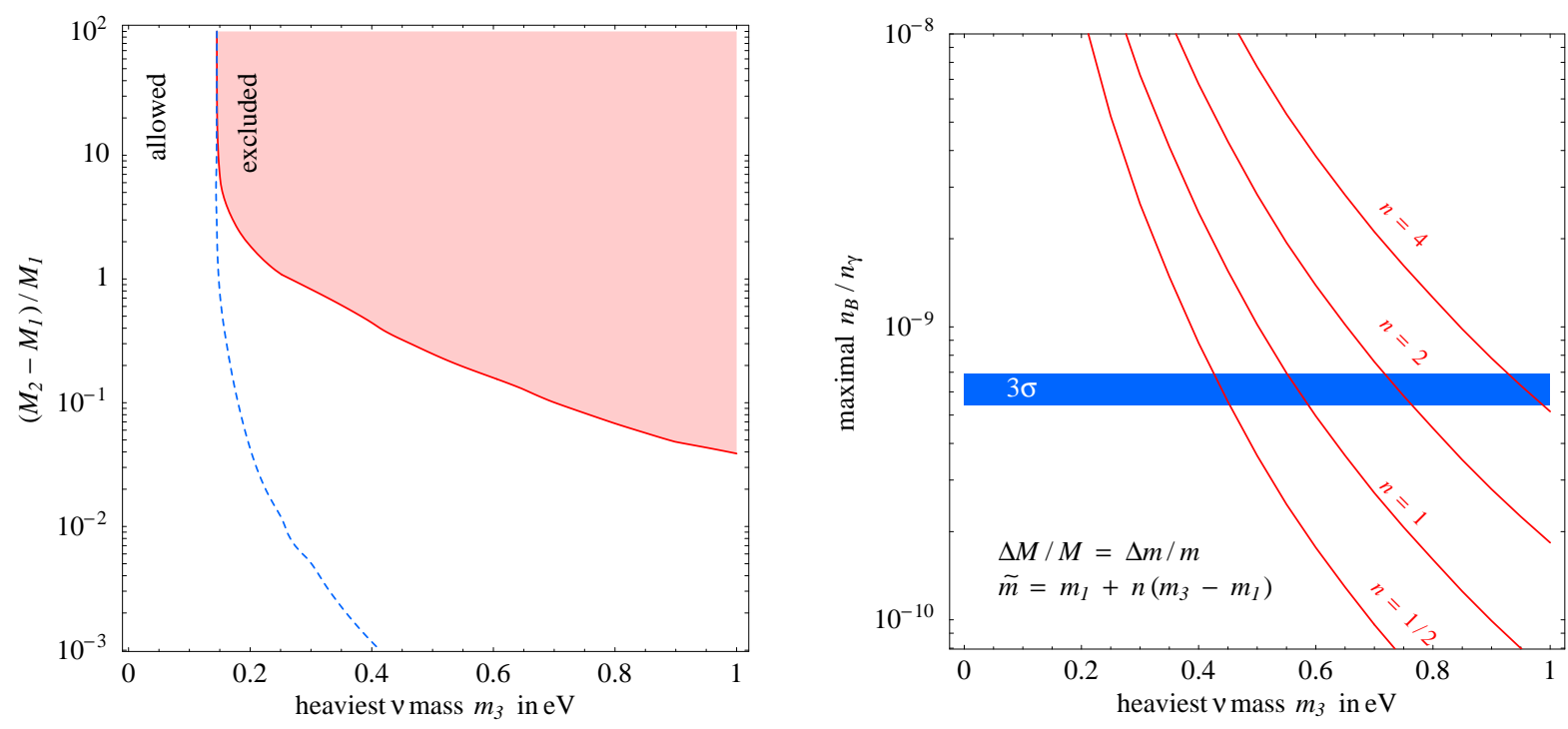

Figure 4: In fig. 纬 we estimate how much the maximal value of neutrino mass compatible with thermal leptogenesis increases when right-handed neutrinos are allowed to be quasi-degenerate. The dashed line includes only the resonant enhancement of CP-violation, eq. (19), while the continuous line includes all effects. A numerical sampling confirms that these constraints can be saturated and even slightly exceeded. Fig. 43 holds in models where everything is as degenerate as neutrinos, see eq.s (20). The parameter $n$ quantifies how much $\tilde{m}_{i}$ are assumed to be close to neutrino masses $m_{i}$. As $n$ increases our assumptions get relaxed, and therefore the constraint on $m_{3}$ becomes weaker.

i.e. $M_{1} \simeq M_{2}=M_{3}$ and assuming $\Gamma_{2}=\Gamma_{3}$. In such a special case $S_{2}=S_{3}$ and the asymmetry reduces to:

$$
\left|\varepsilon_{1}\right|=\frac{M_{1}}{M_{2}} \frac{\Gamma_{2}}{M_{2}} S_{2}\left|I_{2}+I_{3}\right|<\frac{1}{8 \pi} \frac{M_{1}}{v^{2}}\left(m_{3}-m_{1}\right) S_{2} .
$$

At the resonance this gives

$$
\left|\varepsilon_{1}\right|=\frac{1}{2} \frac{M_{1}}{M_{2}}\left|I_{2}+I_{3}\right| \simeq \frac{1}{2}\left|I_{2}+I_{3}\right|<\frac{1}{2} \frac{\left(m_{3}-m_{1}\right)}{\tilde{m}_{2}} .
$$

As a result the asymmetry is smaller than in the previous 2 quasi degenerate right-handed neutrino case by one power of the orthogonality factor that suppresses $I_{2}+I_{3}$. In other words $\varepsilon_{1}$ is enhanced by the resonance factor but still suppressed when neutrinos are quasi-degenerate, by the $\left(m_{3}-m_{1}\right) / \tilde{m}_{2}=$ $\Delta m_{\text {atm }}^{2} / \tilde{m}_{2}\left(m_{3}+m_{1}\right)$ factor already discussed above.

The constraint on neutrino masses obtained in this special case is shown by the dashed line in fig. 田 and is significantly stronger than the one obtained in the previous case (continuous line). This special case, which does not correspond to the most conservative situation, roughly corresponds to what is obtained in 3. In this reference, the hierarchical asymmetry has been enhanced by the resonance factor but was still suppressed by the orthogonality factor (which is $\sim 10^{-3}$ for $m_{1} \sim 1 \mathrm{eV}$ ).

\section{The most realistic case}

Since the leptogenesis constraint on neutrino masses is relevant only for a quasi-degenerate spectrum of light neutrinos, one can wonder which is the most natural right-handed neutrino mass spectrum that produces quasi-degenerate neutrinos. Presumably the answer is: three quasi-degenerate righthanded neutrinos. In fact, other spectra (e.g. hierarchical right-handed neutrinos) can give rise to quasi-degenerate neutrinos only in presence of an appropriate precise correlation between the Yukawa 
couplings and the right-handed neutrino masses. It is difficult to find a theoretical reason that can justify this kind of correlation among different objects.

With three quasi-degenerate right-handed neutrinos, no qualitatively new effect appears with respect to the 'two neutrino' quasi-degenerate case of eq. (14) we discussed above. An orthogonality suppression similar to the one of the special case above is generically not present. As a consequence the constraint on neutrino masses is again well estimated by the continuous line in fig. 固.

There exists one specific pattern, which is probably the most realistic one, which leads to more stringent bounds. In fact, if neutrinos were quasi-degenerate, the degeneracy would presumably not be accidental but due to some reason: a broken $\mathrm{SO}(3)$ flavour symmetry is probably the simplest possibility. One expects that in such framework all quantities, and not only neutrino masses, are close to the ideal limit where three degenerate right-handed neutrinos give equal masses (with equal CP phases) to three orthogonal combinations of left-handed neutrinos. Therefore one expects something like $\tilde{m}_{i}-\tilde{m}_{j} \approx m_{i}-m_{j}$ and

$$
\begin{aligned}
& \frac{M_{2}-M_{1}}{M_{1}} \sim \frac{\tilde{m}_{2}-\tilde{m}_{1}}{\tilde{m}_{1}} \sim \frac{m_{2}-m_{1}}{m_{1}} \approx \frac{\Delta m_{\mathrm{sun}}^{2}}{2 m_{1}^{2}} \approx 0.510^{-4}\left(\frac{\mathrm{eV}}{m_{2}}\right)^{2}, \\
& \frac{M_{3}-M_{2}}{M_{2}} \sim \frac{m_{3}-m_{2}}{m_{2}} \sim \frac{\tilde{m}_{3}-\tilde{m}_{2}}{\tilde{m}_{2}} \approx \frac{\Delta m_{\mathrm{atm}}^{2}}{2 m_{3}^{2}} \approx 10^{-3}\left(\frac{\mathrm{eV}}{m_{2}}\right)^{2} .
\end{aligned}
$$

Right-handed neutrinos can be more degenerate than in the above estimates if only the neutrino Yukawa couplings deviate from the symmetric limit, and can be less degenerate only if there are accidental cancellations between non-universal Yukawa couplings and non-degenerate $M_{1,2,3}$ in the see-saw prediction for neutrino masses. Within the parameterization of eq. (2), our reasonable assumption means $\left|y_{i j}\right| \lesssim \Delta m^{2} / m^{2}$.

To calculate the bound on the neutrino mass considering the realistic example of eq.s (20), it is an excellent approximation to take in eq. (4) (and similar equation for $N_{2,3}$ ) all $M_{i}$ equal to the same value $M$ everywhere except in the resonance $S$ factors, and to take all decay widths equal to the same value $\Gamma$ everywhere except in the Yukawa coupling $I$ factors. It is also an excellent approximation to calculate the wash-out effects in the symmetric limits with $\tilde{m}_{1}=\tilde{m}_{2}=\tilde{m}_{3} \equiv \tilde{m}$. Making these approximations (and assuming $M_{1,2,3} \gg 10^{10} \mathrm{GeV}$, so that interaction rates induced by charged lepton Yukawa couplings can be neglected) the complicated set of Boltzmann equations for the lepton asymmetries generated by $N_{1,2,3}$ decays splits into three independent Boltzmann equations. The net result is that the efficiency is the same as in 'one flavour' approximation, with the CP-asymmetry now given by $\varepsilon=\varepsilon_{1}+\varepsilon_{2}+\varepsilon_{3}$ (which can be rewritten in a rephasing-invariant way as a trace of an appropriate matrix function of $\lambda$ and $M_{N}$, see [13]). In this case in the limit $M_{1}=M_{2}$ the asymmetry reduces to the one of the special case above which is orthogonality suppressed by factors $\left(m_{3}-m_{1}\right) / \tilde{m}_{i}$. Therefore the only terms which are not orthogonality suppressed and which are dominant are the terms involving the $M_{2}^{2}-M_{1}^{2}$ splitting. Neglecting higher order terms in the splitting parameters $\left(M_{3}-M_{1}\right) / M_{1},\left(M_{2}-M_{1}\right) / M_{1}$ and $\left(M_{2}-M_{1}\right) /\left(M_{3}-M_{1}\right)$ these terms are:

$$
\varepsilon \simeq-2 \frac{\Gamma}{M} S_{2} I_{2}+2 \frac{\Gamma}{M} S_{3} I_{3} \frac{M_{2}-M_{1}}{M_{3}-M_{1}} \frac{\left(M_{3}-M_{1}\right)^{2}-\Gamma^{2} / 4}{\left(M_{3}-M_{1}\right)^{2}+\Gamma^{2} / 4} .
$$

The first term is the asymmetry of the 2 quasi degenerate case of eq. (14) (from both $\varepsilon_{1}$ and $\varepsilon_{2}$ ) in which $N_{3}$ has a negligible effect. The second term comes from the difference between the $N_{1}-N_{3}$ and $N_{2}-N_{3}$ mass splittings in the diagram involving these right-handed neutrinos. It is easy to check that although this term can have large effects for large $M,{ }^{5}$ it has a completely negligible effect on the bound which is obtained for smaller $M$. The asymmetry relevant for the determination of the bound reduces therefore to the 2 quasi-degenerate case above of eq. (14) summed on both $\varepsilon_{1}$ and $\varepsilon_{2}$. In addition to the fact that it is not suppressed by any orthogonality relation factor, it turns out to be little suppressed by the resonance factor $S_{2}$. For values of $m_{1}$ around $\mathrm{eV}$ and with $\tilde{m}_{j} \sim m_{i}$ the factor $S_{2}$ is naturally

\footnotetext{
${ }^{5}$ At large $M$ (e.g. above $10^{13 \div 14} \mathrm{GeV}$ ), unlike for smaller $M$, the sum of both terms is suppressed by orthogonality $\left(m_{3}-m_{1}\right) / \tilde{m}_{i}$ factors, and the corresponding neutrino mass bound is therefore suppressed.
} 
at the resonance or close to it. From eq. (20a), the resonance condition $\Gamma \sim 2\left(M_{2}-M_{1}\right)$ gives:

$$
M \simeq 10^{11} \mathrm{GeV}\left(\frac{\mathrm{eV}}{m_{i}}\right)^{3},
$$

The only large suppression can come from the factor $I_{2}$ for values of the $\tilde{m}_{i}$ close to $m_{1}$. From the bound on $I_{2}$ given in appendix $\mathrm{B}$ this suppression goes like:

$$
\left|\varepsilon_{\max }\right|=I_{2}^{\max } \simeq\left(1-m_{1} / \tilde{m}\right)^{3 / 2},
$$

where for simplicity in the last equality we have taken all $\tilde{m}_{i}$ equal to the same value $\tilde{m}$. Using this bound, in fig. $4 \mathrm{~b}$ we give the baryon asymmetry we obtain as a function of $m_{3}$ for values of $\tilde{m}=m_{1}+n\left(m_{3}-m_{1}\right)$ with $n=\{1 / 2,1,2,4\}$. Taking $\tilde{m}=m_{3}(n=1)$, as the generic example for the case that the $\tilde{m}$ would be precisely of order the neutrino masses, gives the constraint

$$
m_{3}<0.6 \mathrm{eV}
$$

which is stronger than in the conservative case because we are now assuming smaller $\tilde{m}$, close to neutrino masses. Taking larger values of $\tilde{m}$ leads rapidly to larger bounds. For example taking $n=4$ (which starts be fine-tuned) gives $m_{3}<1 \mathrm{eV}$. The dependence of the bound on $M_{1}$ is quite sensitive to the exact value of the splitting we take for the right-handed neutrinos because this determines the position of the resonance. For eq.s (20) the bound is obtained for values close to where the $N_{1} / N_{2}$ resonance occurs, i.e. around $M \sim 10^{11} \mathrm{GeV}$. Without a predictive flavour model which would show how the correlations between the seesaw parameters at the origin of the degenerate spectrum occur, these bounds obtained from eq.s (20) are only indicative of what happens and in order to have a safe bound we must consider the conservative case of fig. 4 a (where $n$ was left as a free parameter in order to maximize the asymmetry, so that $\tilde{m}_{j}$ can differ from $m_{i}$ by factors of order one). Even in a very constrained situation the neutrino masses can be as large as $0.6 \mathrm{eV}$, eq. (24). ${ }^{6}$

\section{Leptogenesis in alternative minimal models of neutrino masses}

Generic neutrino masses can be mediated by tree-level exchange of:

a) At least three fermion singlets ('right-handed neutrinos'), described by the see-saw Lagrangian of eq. (11).

b) At least three fermion $\mathrm{SU}(2)_{L}$ triplets with zero hypercharges: the Lagrangian keeps the same structure as in the singlet case, but with different contractions of the $\mathrm{SU}(2)_{L}$ indices that we explicitly show:

$$
\mathscr{L}=\mathscr{L}_{\mathrm{SM}}+\bar{N}_{i} i \not D N_{i}+\left(\lambda^{i j} \tau_{\alpha \beta}^{a} N_{i}^{a} L_{j}^{\alpha} H^{\beta}+\frac{M_{N}^{i j}}{2} N_{i}^{a} N_{j}^{a}+\text { h.c. }\right) .
$$

The index $a$ runs over $\{1,2,3\}, \alpha, \beta$ over $\{1,2\}$ and $\tau^{a}$ are the Pauli matrices.

c) One scalar ('Higgs') triplet $T$ with appropriate hypercharge, such that the most generic renormalizable Lagrangian is

$$
\mathscr{L}=\mathscr{L}_{\mathrm{SM}}+\left|D_{\mu} T\right|^{2}-M_{T}^{2}|T|^{2}+\left(\lambda_{T}^{i j} L^{i} L^{j} T+M H H T^{*}+\text { h.c. }\right) .
$$

Neutrino masses can be also mediated by combinations of the above possibilities, among which it is interesting to consider:

\footnotetext{
${ }^{6}$ Stronger constraints will arise if supersymmetry exists and if right-handed neutrinos lighter than in eq. (22) will be needed to avoid gravitino overproduction [12. Furthermore, making extra 'reasonable' assumptions about the flavour structure of Yukawa couplings one gets a smaller CP-asymmetry (suppressed by 2 powers of the quasi-degeneracy factor, rather than by $3 / 2$ powers) and consequently a slightly stronger constraint on neutrino masses.
} 

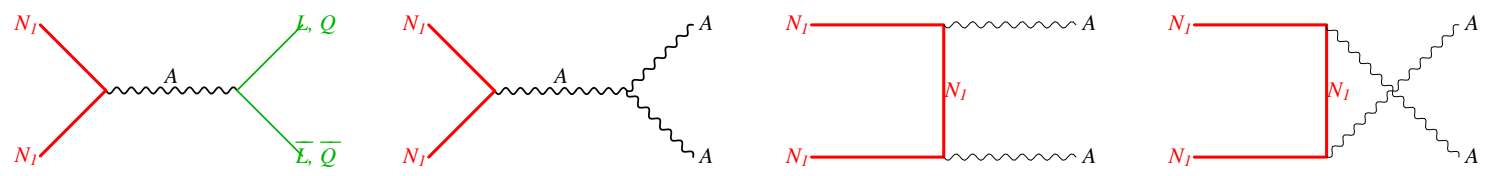

Figure 5: Fermion triplet leptogenesis. Feynman diagrams that give the new interaction rate $\gamma_{A}$.

d) Two or more scalar triplets $T$ with similar interactions.

e) One scalar triplet and fermion singlets.

Model c) has the minimal number of beyond-the-SM parameters $(8+3$, while a) and b) have both $12+6$ extra parameters) but does not lead to a large enough lepton asymmetry. ${ }^{7}$ Adding to c) other scalar triplets as in d) or fermion singlets as in e) allows successful leptogenesis ${ }^{8}$ at the price of introducing more unknown parameters than in a), b) and c). Theoretically, case a) is preferred because singlets nicely marry with grand unification (which is maybe the most promising speculation that we have today). The combination e) can also find theoretical support because 3 singlets and a Higgs triplet are naturally present in renormalizable $\mathrm{SO}(10)$ models (as well as the in underlying left-right models) and can play an important rôle in leptogenesis [18, 19, [20]. This possibility does not lead to relevant constraints on neutrino mass. Cases b) and d) seem to be less natural within a grand unified scheme. E.g. fermion triplets with the needed Yukawa couplings can arise from adjoints of SU(5). However we believe that it is worth to study case b) because it is the only possibility which, with as few parameters as the singlet model, can lead to successful leptogenesis. This is what we show in the following.

Neutrino singlets trivially allow thermal leptogenesis: not having gauge interactions they easily satisfy the out-of-equilibrium Sacharov condition for baryogenesis. Fermion triplets (as scalar triplets) have gauge interactions so that it is more difficult to have a non thermal abundancy. We now study thermal leptogenesis in decays of charged particles, finding that gauge interactions, rather than preventing baryogenesis, make it more predictive. The point is that gauge interactions involve two particles (see fig. [5] and are therefore doubly Boltzmann suppressed at temperatures below their mass (fig. 6a shows an explicit example), so that they cannot wash-out the lepton asymmetry in an efficient way. ${ }^{9}$ On the contrary gauge interactions are efficient at higher temperatures and thermalize the initial abundancy, so that the final baryon asymmetry almost never depends on it (unlike what happens in the singlet case).

With fermion triplets neutrino masses are still given by the usual see-saw formula, $m=-v^{2} \lambda^{T}$. $M_{N}^{-1} \cdot \lambda$, without changing any $\mathcal{O}(1)$ factor. Using the same notations as in the singlet case (see eq. (4) for a more precise discussion) the $\mathrm{CP}$ asymmetry is now given by

$$
\varepsilon_{1}=\sum_{j=2,3} \frac{3}{2} \frac{M_{1}}{M_{j}} \frac{\Gamma_{j}}{M_{j}} I_{j} \frac{V_{j}-2 S_{j}}{3},
$$

and is therefore 3 times smaller in the hierarchical limit. The final amount of baryon asymmetry is given by the CP-asymmetry times the efficiency factor $\eta$ times a numerical coefficient which is 3 times bigger than in the singlet case because now $N_{1}$ has three components:

$$
\frac{n_{B}}{n_{\gamma}}=-0.029 \varepsilon_{1} \eta
$$

The $N_{1}$ decay width is given by the same expression as in the singlet case, so that the thermally averaged decay rate $\gamma_{D}$ becomes 3 times bigger (again because $N_{1}$ now has 3 components). The on-shell part

\footnotetext{
${ }^{7}$ One expects that a CP asymmetry in the total triplet decay rate, $\Gamma(T \rightarrow L L) \neq \Gamma\left(T^{*} \rightarrow \bar{L} \bar{L}\right)$ arises at two or more loops. Taking into account how $\lambda_{T}$ and the Yukawa couplings of charged leptons break $\mathrm{U}(3)_{L} \otimes \mathrm{U}(3)_{E}$ flavour rotations and proceeding along the lines of [15] we find that a non zero CP asymmetry needs four powers of $\lambda_{\tau}$ and two powers of $\lambda_{\mu}$, and is therefore too small (unless enhanced by IR effects, which might give only a mild logarithmic GIM-like suppression).

${ }^{8}$ Leptogenesis in case d) has been studied in [16] 17. We do not consider further this possibility here.

${ }^{9} \mathrm{~A}$ similar result was found for scalar triplets in 17 .
} 

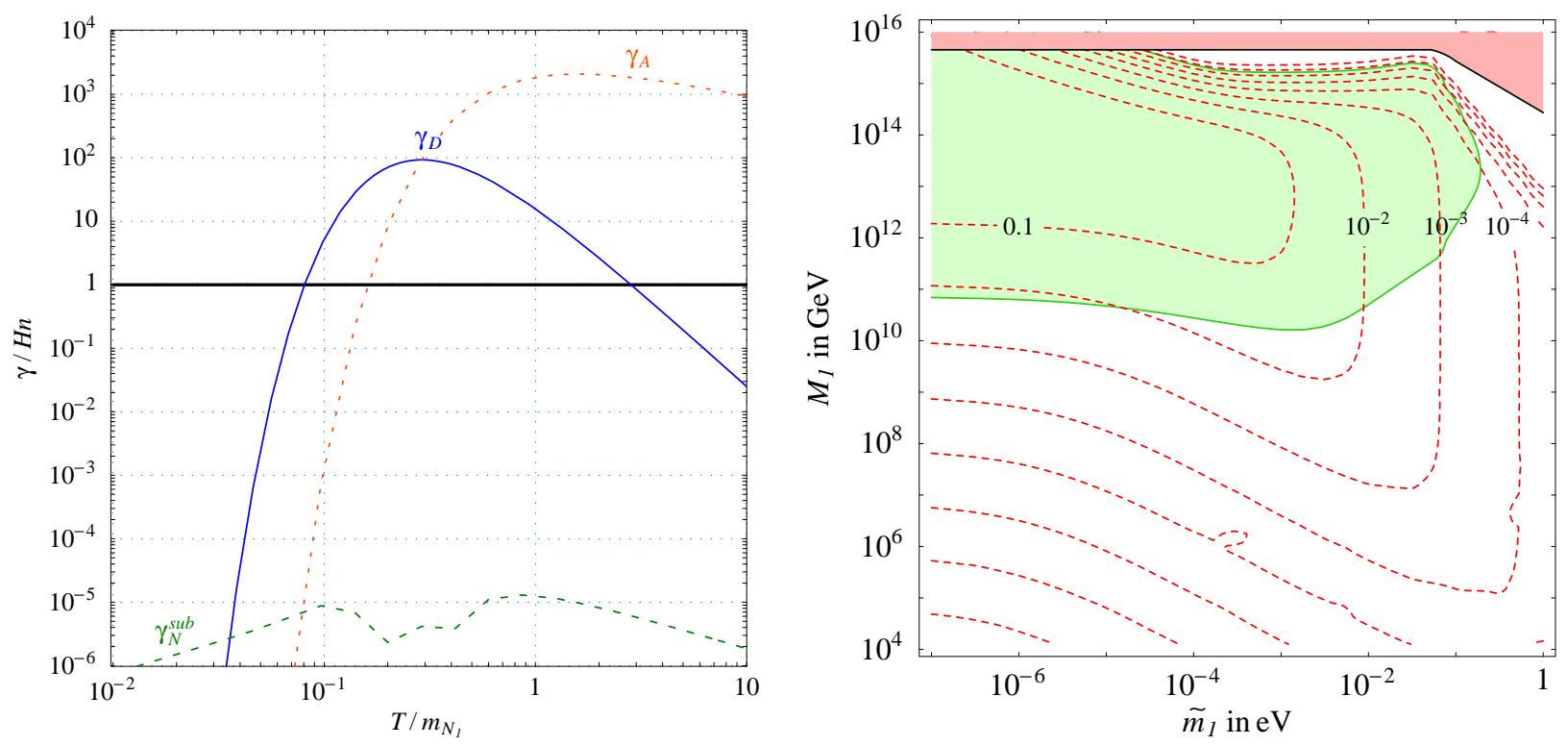

Figure 6: Fermion triplet leptogenesis. Fig. 6 a: Interaction rates $\left|\gamma / H n_{\gamma}\right|$ for $M_{1}=10^{10} \mathrm{GeV}$ and $\tilde{m}_{1}=0.06 \mathrm{eV}$. Fig. [6b: contour-levels of the efficiency $\eta$. Successful leptogenesis with (infinitely) hierarchical triplets is possible inside the green area.

of $\Delta L=2$ scattering rates, equal to $\gamma_{D} / 4$ [4, becomes therefore also 3 times bigger, and the off-shell part is affected in a different way. We find:

$$
\begin{aligned}
\hat{\sigma}_{N s}\left(L H \rightarrow \bar{L} H^{*}\right)= & \frac{\left(\lambda \lambda^{\dagger}\right)_{11}^{2}}{4 \pi}\left[2+x D_{s}^{2 \text { sub }}+(2-3 x \xi) \operatorname{Re} D_{s}+3 \xi(x \xi-2)-\right. \\
& \left.-\frac{2 \ln (1+x)}{x}\left(1-\left(\operatorname{Re} D_{s}-3 \xi\right)(1+x)\right)\right] \\
\hat{\sigma}_{N t}\left(L L \rightarrow H^{*} H^{*}\right)= & \frac{\left(\lambda \lambda^{\dagger}\right)_{11}^{2}}{2 \pi}\left[\frac{3 x}{2}\left(\xi^{2}+\frac{2}{1+x}\right)+\left(3 \xi-\frac{3}{2+x}\right) \ln (1+x)\right]
\end{aligned}
$$

where $x=s / M_{1}^{2}$. A 'natural' value of the parameter $\xi$ is $\xi=m_{3} / \tilde{m}_{1}$. It is defined as follows: the amplitude of $N_{2,3}$-mediated scatterings is written as $\xi$ times the value computed assuming that $N_{2,3}$ give the same neutrino masses as $N_{1}$. In order to deal with this issue in a more precise way one should know the flavour structure of $N_{1}$ couplings and solve Boltzmann equations for the asymmetries in the various flavours. Our simplified approach is justified by the fact that $\xi$ has a minor impact in most of the 'reasonable' parameter space. The reduced cross sections $\hat{\sigma}$ are related to the corresponding interaction rates as summarized in 4, that also explains how to perform a proper subtraction of the $s$-channel propagator $D_{s}$.

We computed gauge scatterings $\hat{\sigma}_{A}$ (see fig. 5) summing over the $12 \mathrm{SM}$ fermionic doublets $D=$ $\left\{L_{1,2,3}, Q_{1,2,3}\right\}$ and neglecting scatterings into Higgs doublets (since they are not enhanced by a large number of final states and since the threshold behavior at $s \simeq M_{1}^{2}$ is the same). At $s \gg M_{1}^{2}$ the $N N \rightarrow A A$ cross section is enhanced by IR effects. We find:

$$
\hat{\sigma}_{A}\left(N_{1} N_{1} \rightarrow D \bar{D}, A A\right)=\frac{6 g_{2}^{4}}{\pi}\left(1+\frac{2}{x}\right) r+\frac{2 g_{2}^{4}}{\pi}\left[-r\left(4+\frac{17}{x}\right)+3\left(1+\frac{4}{x}-\frac{4}{x^{2}}\right) \ln \frac{1+r}{1-r}\right]
$$

where $r=\sqrt{1-4 / x}$. Symmetry factors for initial and final state particles are included in the reduced cross sections. For simplicity we neglected $\Delta L=1$ scatterings, three body decays, one loop and thermal corrections. 
The Boltzmann equation involve the new gauge term, $\gamma_{A}$ :

$$
\begin{aligned}
s H z \frac{d Y_{N_{1}}}{d z} & =-\left(\frac{Y_{N_{1}}}{Y_{N_{1}}^{\mathrm{eq}}}-1\right) \gamma_{D}-\left(\frac{Y_{N_{1}}^{2}}{Y_{N_{1}}^{2 \mathrm{eq}}}-1\right) \gamma_{A}, \\
s H z \frac{d Y_{\mathcal{B}-\mathcal{L}}}{d z} & =-\gamma_{D} \varepsilon_{N_{1}}\left(\frac{Y_{N_{1}}}{Y_{N_{1}}^{\mathrm{eq}}}-1\right)-\frac{Y_{\mathcal{B}-\mathcal{L}}}{Y_{L}^{\mathrm{eq}}}\left(\frac{\gamma_{D}}{2}+2 \gamma_{N}^{\mathrm{sub}}\right),
\end{aligned}
$$

where $s$ is the entropy of SM particles, $H$ is the Hubble constant at temperature $T$.

The efficiency factor $\eta\left(\tilde{m}_{1}, M_{1}, \xi\right)$, computed solving numerically the Boltzmann equations, is shown in fig. [b assuming the reasonable value of $\xi$ (other reasonable values would give minor differences). The result is qualitatively different from the analogous result in the singlet case, shown in fig. 8 of [4]

- At $\tilde{m}_{1} \ll 10^{-3} \mathrm{eV}$ only gauge interactions drive the $N_{1}$ abundancy close to thermal equilibrium (unless $M_{1} \gtrsim 10^{15} \mathrm{GeV}$ ). A 'relic' fraction of $N_{1}$ survives to gauge annihilations and later decays generating the baryon asymmetry with efficiency $\eta \approx M_{1} / 10^{13} \mathrm{GeV}\left(\eta\right.$ is larger if $\tilde{m}_{1} \sim 10^{-3} \mathrm{eV}$ because some $N_{1}$ decay during the annihilation stage). Gauge interactions give a stronger suppression at smaller $M_{1}$, because at low temperatures the expansion of the universe is slower, $H \sim T^{2} / M_{\mathrm{Pl}}$. This can be contrasted to what happens in the $\nu_{R}$ case: since it has no gauge interactions the efficiency $\eta$ only has a minor dependence on $M_{1}$.

- At $\tilde{m}_{1} \gg 10^{-3} \mathrm{eV}$ neutrino Yukawa interactions drive the $N_{1}$ abundancy close to thermal equilibrium. As a consequence there are only $\mathcal{O}(1)$ differences between singlet and triplet leptogenesis.

Assuming a sufficiently huge hierarchy, $M_{1} \gg M_{2,3}$ we can derive a region where thermal leptogenesis can be successful using our maximal CP-asymmetry (which is $1 / 3$ of what obtained in eq. (11) in the singlet case). The lower bound on the $N_{1}$ mass and the upper bound on neutrino masses are at $3 \sigma$

$$
M_{1} \gtrsim 1.510^{10} \mathrm{GeV} \quad m_{3}<0.12 \mathrm{eV} .
$$

These bounds are slightly stronger than in the right-handed neutrino case ${ }^{10}$, and are subject to all the caveats discussed in that case. In particular quasi-degenerate $N_{i}$ allow leptogenesis at the TeV-scale: fig. 6] shows that, despite gauge interactions, the efficiency remains large enough. This case is testable at collider, where $N_{i}$ triplets can be produced and detected (while $N_{i}$ singlets cannot, because have too low cross sections).

Only $\mathcal{O}(1)$ factors are modified if supersymmetry is introduced in the usual minimal way.

\section{Conclusions}

Atmospheric oscillations suggest that the heaviest neutrino mass $m_{3}$ is larger than about $0.05 \mathrm{eV}$. Various techniques could reach the necessary sensitivity and presently give the following 95\% C.L. bounds: $m_{3}<2.2 \mathrm{eV}$ from $\beta$-decay $21, m_{3}<1.0 h \mathrm{eV}$ from neutrino-less double-beta decay 22 (assuming Majorana masses; $h \approx 1$ renormalizes the uncertain nuclear matrix element), $m_{3}<0.23 \mathrm{eV}$ from cosmology 23] (assuming a minimal model). A stronger constraint, $m_{3} \lesssim 0.1 \mathrm{eV}[\underline{3}$, is obtained assuming thermal leptogenesis within see-saw models with hierarchical right-handed neutrinos. We reanalyzed this leptogenesis constraint, merging the revised computation of dynamics of leptogenesis of 4 with a revised bound on the CP-asymmetry (see eq. (11)). It is weaker than previous bounds, and its validity needs extra assumptions to discard a special (but non necessarily fine-tuned) choice of parameters that can give a much larger asymmetry (see eq. (10) and fig. 10. Furthermore in appendix $\mathrm{A}$ we explained why and which single Boltzmann equation for the total $B-L$ asymmetry is a good approximation in the region where the constraint on neutrino masses is saturated.

\footnotetext{
${ }^{10}$ Within one order of magnitude, our bound on $M_{1}$ is in agreement with the estimated bound in [17] for scalar triplets (see also [19).
} 
From this revised analysis we obtain that if neutrinos turn out to be lighter than

$$
m_{i}<0.15 \mathrm{eV}
$$

thermal leptogenesis can generically produce the observed baryon abundancy. This critical value is the present $3 \sigma$ bound (see fig. [3) and can mildly shift with more accurate measurements of $\Delta m_{\mathrm{atm}}^{2}$, of $n_{B}$, or if supersymmetry will be discovered.

We studied what happens dropping the dubious assumption that hierarchical right-handed neutrinos give quasi-degenerate neutrino masses. If neutrinos are heavier than $0.15 \mathrm{eV}$ quasi-degenerate $\nu_{R}$ would be suggested by good taste and allow to weaken largely the leptogenesis constraint. How much depends on why neutrinos are quasi-degenerate. We consider two possible classes of interpretations:

a) Neutrinos are not controlled by any flavour symmetry: this naturally gives large mixing angles and comparable neutrino masses, which might accidentally show some mild level of quasi-degeneracy. If we therefore assume $m_{i} \approx \tilde{m}_{j}$ we find that a mild degeneracy, $\left(M_{2}-M_{1}\right) / M_{1} \sim 0.1$ is sufficient to push the leptogenesis constraint above $1 \mathrm{eV}$ (continuous line in fig. 目). This happens for two different reasons: the CP asymmetry can be resonantly enhanced and is no longer suppressed by one power of the orthogonality suppression factor $\Delta m_{\mathrm{atm}}^{2} / m_{3}^{2}$, see eq. (15). A sub-eV leptogenesis constraint would survive only if one of these two suppressions were present (dashed line in fig. 固 and previous analyses [3]), but this has no reason to generically occur.

b) Some flavour symmetry (e.g. $\mathrm{SO}(3)$ ) keeps left and right-handed neutrinos quasi-degenerate giving $\tilde{m}_{j}$ very close to neutrino masses $m_{i}$. We find that this gives a CP-asymmetry suppressed by $3 / 2$ powers of the quasi degeneracy factor $1-m_{1} / \tilde{m}_{j} \sim \Delta m_{\text {atm }}^{2} / m_{3}^{2}$, see eq. (23), resulting in a constraint $m_{3} \lesssim 0.6 \mathrm{eV}$ (which can be largely relaxed if the $\tilde{m}_{j}$ are slightly larger than the $m_{i}$, see fig. 目).

In the last section we studied leptogenesis in alternative minimal models. Neutrino masses can be mediated by tree-level exchange of right-handed neutrinos, or of fermion $\mathrm{SU}(2)_{L}$ triplets or of scalar triplets. We find that in the last two cases leptogenesis can proceed enough out-of-equilibrium, despite the new effect of gauge interactions. The reason is that their rates are strongly Boltzmann suppressed in the last stages of decay processes. While fermion triplets lead to successful leptogenesis (giving only slightly stronger constraints than with singlets, eq. (31) ) using only a single scalar triplet it seems impossible to achieve a sufficiently large CP-asymmetry.

Acknowledgments This work has been partially supported by the EU under TMR contracts HPRNCT-2000-00148, HPRN-CT-2000-00152 and for T.H. by the EU Marie Curie contract HPMF-CT01765. We thank Sacha Davidson for useful suggestions and painful criticisms.

\section{A Boltzmann equations with flavour}

We here explain how the full network of Boltzmann equations can be approximated with a single equation for the total $B-L$ asymmetry when computing the constraint on quasi-degenerate neutrino masses. In the standard approximation one writes one Boltzmann equation for the total asymmetry, without caring about how it is shared among different lepton doublet flavours. In simple cases this can be a good approximation [14] if done properly, as the following example shows. Let us suppose that $N_{1}$ decays generate a lepton asymmetry in $\nu_{1}=\left(\nu_{\mu}+\nu_{\tau}\right) / \sqrt{2}$ and that there are wash-out interactions acting on $\nu_{2}=\left(\nu_{\mu}-\nu_{\tau}\right) / \sqrt{2}$ : one can wonder if they are weighted by a) $\left|\left\langle\nu_{1} \mid \nu_{\mu, \tau}\right\rangle\right|^{2}=1 / 2$ or by b) $\left|\left\langle\nu_{1} \mid \nu_{2}\right\rangle\right|^{2}=0$ ? The answer is a) when scatterings induced by the $\tau$ Yukawa coupling are much faster than the expansion of the universe (because they convert $\nu_{1}$ into a incoherent mixture of $\nu_{\mu}$ and $\nu_{\tau}$ ) and b) when they are much slower (because $\nu_{1}$ remains a coherent superposition of $\nu_{\mu}$ and $\nu_{\tau}$ ). Around the 
values $T \sim M_{1} \gg 10^{11} \mathrm{GeV}$ for which the leptogenesis constraint is saturated, all SM lepton Yukawa couplings can be neglected (case b) and the Boltzmann equation for leptogenesis is [14]

$$
\frac{d \rho}{d t}=z s H \frac{d \rho}{d z}=\gamma_{D}\left(1-\frac{Y_{N_{1}}}{Y_{N_{1}}^{\mathrm{eq}}}\right) \frac{\Gamma \Pi-\bar{\Gamma} \bar{\Pi}}{\Gamma+\bar{\Gamma}}-\frac{\left\{\gamma_{N}, \rho\right\}}{8 Y_{L}^{\mathrm{eq}}},
$$

where $\rho$ is the $3 \times 3$ matrix density that fully describes how the 3 flavours share the $B-L$ asymmetry. $\Pi$ $(\bar{\Pi})$ is the projector over the lepton (anti-lepton) flavour to which $N_{1}$ decays with decay width $\Gamma(\bar{\Gamma})$. At tree level $\Gamma=\bar{\Gamma}=\Gamma_{1} / 2$ and $\Pi_{i j}=\bar{\Pi}_{i j}=\left(\Pi_{1}\right)_{i j} \equiv \lambda_{1 i} \lambda_{1 j}^{*} /\left|\lambda \lambda^{\dagger}\right|_{11}$. At one-loop $N_{1}$ decays into leptons and anti-leptons with different rates (giving the total CP-asymmetry $\varepsilon_{1}=(\Gamma-\bar{\Gamma}) /(\Gamma+\bar{\Gamma})$ ) and into different flavours $(\Pi \neq \bar{\Pi}) . \hat{\gamma}_{N}$ is the $3 \times 3$ flavour matrix of interaction rates of $\Delta L=2$ scatterings. It can be decomposed as $\hat{\gamma}_{N}=4 \gamma_{D} \Pi_{1}+\hat{\gamma}_{N}^{\text {sub }}$, where the first term takes into account resonant scatterings mediated by on-shell $N_{1}$, and $\hat{\gamma}_{N}^{\text {sub }}$ describes off-shell scatterings mediated by $N_{1,2,3}$. For all the other symbols we adopted the notations of [4] (e.g. $z=M_{1} / T, \gamma_{D}$ is the decay interaction rate,...).

In general, without making approximations the matrix equation (33) cannot be replaced by a single equation for the total asymmetry $Y_{B-L}=\operatorname{Tr} \rho$, nor by three equations for the diagonal components of $\rho$ (in some flavour basis). In the present case, taking into account that for quasi-degenerate neutrinos $\hat{\gamma}_{N}^{\text {sub }}$ is a linear combination of $\Pi_{1}$ and of $1-\Pi_{1},{ }^{11}$ it is non trivial to verify that at leading order in $\varepsilon_{1}$ the solution to (33) is

$$
\rho=Y_{B-L}\left(\Pi_{1}+\frac{\Pi-\bar{\Pi}}{2 \varepsilon_{1}}\right),
$$

where $Y_{B-L}$ satisfies the Boltzmann equation of [4] in 'one flavour' approximation,

$$
z s H \frac{d Y_{B-L}}{d z}=\varepsilon_{1} \gamma_{D}\left(1-\frac{Y_{N_{1}}}{Y_{N_{1}}^{\mathrm{eq}}}\right)-\frac{Y_{B-L}}{Y_{L}^{\mathrm{eq}}}\left(\frac{\gamma_{D}}{2}+2 \gamma_{N}^{\mathrm{sub}}\right),
$$

that is therefore adequate for studying the heaviest neutrino mass compatible with leptogenesis. This is the equation we used to calculate the constraint on neutrino masses.

\section{B Maximal CP asymmetry with 2 quasi-degenerate $\nu_{R}$}

In the following we compute the maximum value of $I_{2}$ for fixed value of $\max \left(\tilde{m}_{1}, \tilde{m}_{2}\right)$, which allows to give a bound on the asymmetry both in the 2 quasi-degenerate case, eq. (14), and in the "most realistic case" of eq. (21). Using the parameterization in eq. (2), $I_{2}$ can be written as

$$
I_{2}=\frac{1}{\tilde{m}_{1} \tilde{m}_{2}} \operatorname{Im}\left[\left(R \cdot \operatorname{diag}\left(m_{1}, m_{2}, m_{3}\right) \cdot R^{\dagger}\right)_{12}^{2}\right]
$$

with

$$
\tilde{m}_{1}=\left(R \cdot \operatorname{diag}\left(m_{1}, m_{2}, m_{3}\right) \cdot R^{\dagger}\right)_{11}, \quad \text { and } \quad \tilde{m}_{2}=\left(R \cdot \operatorname{diag}\left(m_{1}, m_{2}, m_{3}\right) \cdot R^{\dagger}\right)_{22} .
$$

Neglecting the solar mass splitting, $I_{2}$ depends on 5 real parameters: the complex angles $z_{13}$ and $z_{23}$ and the imaginary part of the angle $z_{12}$ (the real part of $z_{12}$ cancels out because $m_{1}=m_{2}$ ). However, numerical inspection shows that the maximal value of $I_{2}$ can be obtained for any value of $\operatorname{Re} z_{13}$ and of $\operatorname{Re} z_{23}$ : we can therefore put them to zero, simplifying the expression and reducing the number of the free parameters to 3 . Moreover, for fixed value of $\max \left(\tilde{m}_{1}, \tilde{m}_{2}\right)$ the bound is obtained for $\tilde{m}_{1}=\tilde{m}_{2} \equiv \tilde{m}$. For fixed $\tilde{m}_{1}$ the maximum of $\varepsilon_{1}+\varepsilon_{2}$ is also obtained for $\tilde{m}_{1}=\tilde{m}_{2}$. All this together with eq. (35) gives

$I_{2}^{\max }=2 \max _{z} z \sqrt{1-z}\left(1-\frac{m_{1}}{\tilde{m}}\right)\left(1-\frac{m_{1}^{2}}{\tilde{m}^{2}}\right)^{-1 / 2}\left(1-\frac{\tilde{m}-m_{1}}{\tilde{m}+m_{1}} z\right)^{1 / 2}\left(1+\frac{\tilde{m}-m_{1}}{\tilde{m}+m_{3}} z\right)^{1 / 2}\left(1-\frac{\tilde{m}-m_{1}}{\tilde{m}+m_{3}} z\right)^{-1 / 2}$,

where $z=\left[\cosh \left(2 \operatorname{Im} z_{23}\right)-1\right]\left(\tilde{m}+m_{3}\right) / 2\left(\tilde{m}-m_{1}\right)$ can vary in the interval [0,1]. We now need to maximize the previous expression with respect to $z$. This can be done analytically, but gives a

\footnotetext{
${ }^{11}$ A less accurate approximation is $\left(\hat{\gamma}_{N}^{\text {sub }}\right)_{i j} \approx \gamma_{N}^{\text {sub }} \delta_{i j}$ : when left-handed neutrinos are quasi-degenerate $\gamma_{N}^{\text {sub }}$ is controlled by the average squared neutrino mass (rather than by their sum, which is 3 times larger).
} 
lengthy expression. In the limit $\tilde{m} \gg m_{i}$ the maximum is reached for $z=1 / \sqrt{2}$, while in the opposite limit $\tilde{m} \rightarrow m_{1}$ it is reached for $z=2 / 3$. Since these two values are close (and since functions are almost flat around their maximum), an excellent approximation is obtained by setting $z$ to any of these two numbers. Assuming quasi-degenerate neutrinos, $m_{1} \simeq m_{3}$, one finds a simple expression that interpolates between these two numbers, giving

$$
I_{2}^{\max }=\sqrt{1+\frac{5}{2 r}-\frac{1+(1+8 r)^{3 / 2}}{8 r^{2}}} \approx\left(1-m_{1} / \tilde{m}\right)^{3 / 2}
$$

where $r=\tilde{m}^{2} / m_{1}^{2}$ and the last simple approximation is accurate to better than $10 \%$. So far we didn't put any restriction on $\tilde{m}_{3}$. Requiring in addition $\tilde{m}_{3}=\tilde{m}_{1,2}$ also gives eq. (37) with an accuracy better than $10 \%$. We used therefore eq. (37) for all numerical analyses. Note that $I_{2}^{\max }$ reaches unity asymptotically for large $\tilde{m}$.

\section{References}

[1] M. Fukugita and T. Yanagida, Phys. Lett. 174B (1986) 45.

[2] S. Davidson, A. Ibarra, hep-ph/0202239 The DI value of $\varepsilon_{1}$ was present also in K. Hamaguchi, H. Murayama, T. Yanagida, hep-ph/0109030 An earlier work (14 around eq. (5.1)) presented the DI bound as valid only up to cancellations, in agreement with our present findings.

[3] W. Buchmuller, P. Di Bari, M. Plümacher, Nucl. Phys. B665 (2003) 445 (hep-ph/0302092).

[4] G.F. Giudice, A. Notari, M. Raidal, A. Riotto, A. Strumia, hep-ph/0310123

[5] M. Flanz, E.A. Paschos, U. Sarkar, Phys. Lett. B345 (1995) 248; M. Flanz, E.A. Paschos, U. Sarkar, J. Weiss, Phys. Lett. B389 (1996) 693; L. Covi, E. Roulet, F. Vissani, Phys. Lett. B384 (1996) 169; A. Pilaftsis, Phys. Rev. D56 (1997) 5431; A. Pilaftsis, T.E.J. Underwood, hep-ph/0309342 T. Hambye, Nucl. Phys. B633 (2002) 171.

[6] A. Pilaftsis, Nucl. Phys. B504 (1997) 61.

[7] M. Gell-Mann, P. Ramond and R. Slansky, in Supergravity, edited by P. van Nieuwenhuizen and D. Freedman, (North-Holland, 1979), p. 315; S.L. Glashow, in Quarks and Leptons, Cargèse, eds. M. Lévy et al., (Plenum, 1980, New-York), p. 707; T. Yanagida, in Proceedings of the Workshop on the Unified Theory and the Baryon Number in the Universe, edited by O. Sawada and A. Sugamoto (KEK Report No. 79-18, Tsukuba, 1979), p. 95; R.N. Mohapatra and G. Senjanović, Phys. Rev. Lett. 44, (1980) 912.

[8] R. Foot, H. Lew, X.-G. He and G.C. Joshi, Z. Phys. C44 (1989) 441.

[9] E. Ma, Phys. Rev. Lett. 81 (1998) 1171; E. Ma and D.P. Roy, Nucl. Phys. B644 (2002) 290.

[10] G. Lazarides, Q. Shafi and C. Wetterich, Nucl Phys. B181 (1981) 287; R.N. Mohapatra and G. Senjanović, Phys. Rev. D23 (1981) 165; C. Wetterich, Nucl. Phys. B187 (1981) 343.

[11] J.A. Casas, A. Ibarra, hep-ph/0103065

[12] J. R. Ellis, J. Kim, D. V. Nanopoulos, Phys. Lett. B145, 181 (1984); L. M. Krauss, Nucl. Phys. B227, 556
(1983); M. Yu. Khlopov, A. D. Linde, Phys. Lett. 138B, 265 (1984); J. R. Ellis, D. V. Nanopoulos, K. A. Olive, S.-J. Rey, Astropart. Phys. 4, 371 (1996); M. Bolz, A. Brandenburg, W. Buchmuller, Nucl. Phys. B 606, 518 (2001). R. H. Cyburt, J. Ellis, B. D. Fields, K. A. Olive, Phys. Rev. D 67, 103521 (2003). For a review see M.Yu. Khlopov, 'Cosmoparticle physics', World Scientific, 1999.

[13] S. Davidson, R. Kitano, hep-ph/0312007

[14] R. Barbieri, P. Creminelli, A. Strumia, N. Tetradis, Nucl. Phys. B575 (2000) 61 (hep-ph/9911315).

[15] A. Romanino and A. Strumia, Nucl. Phys. B490 (1997) 204 (hep-ph/9610485).

[16] E. Ma and U. Sarkar, Phys. Rev. Lett. 80 (1998) 5716.

[17] T. Hambye, E. Ma and U. Sarkar, Nucl. Phys. B602 (2001) 23.

[18] P. O'Donnell and U. Sarkar, Phys. Rev. D49 (1994) 2118.

[19] T. Hambye and G. Senjanovic, to appear in Phys. Lett. B, hep-ph/0307237

[20] See also G. Lazarides and Q. Shafi, Phys. Rev. D58 (1998) 071702. For a related model based on inflation see also T. Dent, G. Lazarides and R. Ruiz de Austri, hep-ph/0312033

[21] The Mainz collaboration, Nucl. Phys. Proc. Suppl. 91 (2001) 273; The Troitsk collaboration, Nucl. Phys. Proc. Suppl. 91 (2001) 280.

[22] The $0 \nu 2 \beta$ bound on neutrino masses is obtained by combining $0 \nu 2 \beta$ data, The HeIDELBERG-Moscow collaboration, H.V. Klapdor-Kleingrothaus et al., Eur. Phys. J. A12 (2001) 147 (hep-ph/0103062), with oscillation data: see F. Feruglio, A. Strumia and F. Vissani, Nucl. Phys. B637 (2002) 345 (hep-ph/0201291). and references therein.

[23] The cosmological bound on neutrino masses is obtained by combining WMAP data, C. L. Bennett et al., astro-ph/0302207 with large-scale structure data: see D. N. Spergel et al., astro-ph/0302209 and references therein. This is similar to pre-WMAP analyses, A. Lewis and S. Bridle, Phys. Rev. D66 (2002) 103511. Weaker more conservative bounds are obtained in $\mathrm{S}$. Hannestad, astro-ph/0303076 JCAP03050042003. 